\title{
Secure Internet Banking Authentication
}

\author{
P.Suba, M.Mailsamy, S.Sinduja
}

\begin{abstract}
Check expect an essential improvement in checking any web banking industry, and heaps of banks and various affiliations have since a long time earlier depended on username/puzzle word combos to request clients. Holding usernames and passwords for a huge proportion of records changes into a huge and wasteful undertaking. likewise, inheritance check approachs have tumbling again and again, and that they are not confirmed against a not all that horrible kind of assaults which will be prompted against clients, systems, or endorsing servers. Reliably, data break reports weight that aggressors have made diverse welcome tech strategies to take clients' accreditations, which can cause a liberal risk. During this paper, I will with everything considered propose Associate in Nursing canny and reasonable client bolster subject abuse explicit contraptions that use absolutely amazing science neighborhood people like encoding, automated etching, and hashing. The system edges from the general use of present managing and specific cautious solid and wearable contraptions which will change clients to execute a verified solicitation appear. Our made point doesn't require Associate in Nursing check server to keep up static username and Arcanum tables for trademark and substantiating the validity of the login clients. It not exclusively is secure against question word related strikes, at any rate can in like way confine replay assaults, shoulder-surfing ambushes, phishing ambushes, and data break scenes.
\end{abstract}

Keywords : Security; Authentication; One-Time Username; Access Control.

\section{INTRODUCTION}

Standard check plans like the username/word combo cause an essential risk to the net cash related affiliations, budgetary structures, and their clients. Most present solicitation structures dole out or pull in a client to pick a static and express client id that goes about as a drawing. This static drawing is routinely associated with the client for an expansive time. Unfortunately, clients will all around utilize a proportionate client id in different area and structures. Plus, two or three clients still utilize a dubious word transversely over online records and structures. per a consistent report, $51 \%$ of the checked on clients use a similar word transversely over absolutely abrupt targets, and in excess of seventy seven of the people either somewhat change or use existing passwords with clear misleads.

To show regardless reasonable individual gadgets will improve not just security at any rate conjointly client ace by proposing a one-time username confirmation in like way an ensured check code for each login session. The client

Revised Manuscript Received on August 14, 2019.

P.Suba, PG Scholar, Computer Science and Engineering, Vivekananda College of Engineering for Women, Tiruchengode, India. (E-mail: subhasweety52@gmail.com)

M.Mailsamy, Assistant Professor, Computer Science and Engineering, Vivekananda College of Engineering for Women, Tiruchengode, India. (E-mail: mailsamym@gmail.com)

S.Sinduja, Assistant Professor, Computer Science and Engineering, Vivekananda College of Engineering for Women, Tiruchengode, India. (E-mail: sindujanaga @ gmail.com) shouldn't be compelled to memorise several usernames or recall complicated passwords.

\section{RELATED WORK}

The objectives of this study square measure to style a completely unique authentication scheme victimization dynamic usernames and to decrease the requirement for managing customer's capacities at a bound together territory. Ienvision that the new style ought to refute a few strikes and issues like keylogger ambushes, shoulder-surfing attacks, data break events, Arcanum use, and elective human parts. Keylogger ambushes are getting extra advanced and will target static confirmation plans. A keylogger will be a module gear contraption or a get-together program that goes about as a compromising system annoying the hurt person's pc. The basic goal of maltreatment keyloggers is to catch and watch each keystroke typewritten on the shocking disaster's pc that thoroughly joins insistence information like usernames and unsafe passwords. By and large, keylogger pack and contraption aren't incite to find, particularly on open PCs. Some honest keylogger social event is unmoving in the thing pack and doesn't show up inside the task director strategy

\section{EXISTING SYSTEM}

To look at a pack key understanding drawback wherever a customer isn't generally mindful of his neighbors while the property graph is of line. In our drawback, there's no gathered low-level arranging customers. a social affair key synchronization with these choices is marvelously appropriate for accommodating affiliations.

\subsection{DRAWBACKS}

Secure information sharing among a bunch that counters corporate executive threats of legitimate nevertheless malicious users is a vital analysis issue.

\section{PROPOSED SYSTEM}

Created associate degree actively secure protocol from a passively secure one.

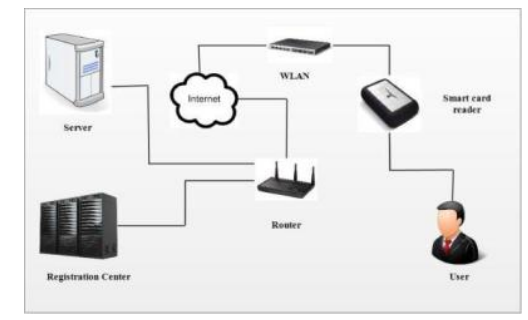

Fig1.1.System Architecure

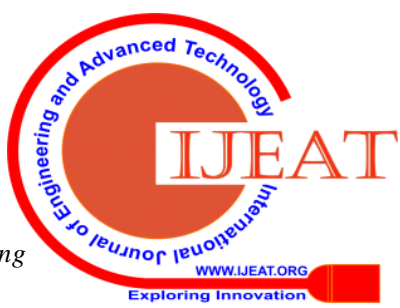


In our work, we have a tendency to failed to think about the way to update the cluster key additional expeditiously than simply running the protocol once more, once user memberships are dynamical

\subsection{ADVANTAGES}

- Secure to share knowledge into the teams.

- Unwanted person or third party can't access cluster communication

\section{MODULES \& RESULTS}

\section{USER INTERFACE DESIGN}

This is the first module for our project. In this User Interface Design we create Registration and Login Page, If you are a new user go to registration page and register your own account, After Registration you will go to login page and login your account, After the login page you will get your own account.

\section{GROUP CREATION}

This is the second module in our project. In this module we create a group in a social network like Facebook, Google+, etc. In this module we create a group for group members like friends. In this Admin creates a group and adds group members to our group. The group members are nothing but friends like college friends, school friends, office friends etc. The group can be created by admin and add group members, the admin have authority to remove the group member and add the group member. The group members have right to exit from the group.

This is the third module of our project. In this module $\mathrm{i}$ generate a group key for group members. The admin creates a group key for group members for security. The admin creates a group for safe sharing of data, images, video, messages etc. The admin generates group key for all group members. The group members are nothing but friends uses this group key for security purpose.

\section{SESSION KEY WITH ENCRYPTION}

This is the fourth module of our project. In this module we create private key for all group members. The admin create this private key for all group members. Every group member has their own identity so we create separate key for all group members. The private key can be generated by admin. The private are nothing but secret key for all group members. The private is like OTP(one time password), The onetime password can be valid for certain period of time like 60 seconds, After 60 seconds it cannot be valid, For this no one couldn't open your messages, images, video etc. The private key generation can be used for security purpose.

\section{SESSION KEY WITH DECRYPTION}

This is the fifth module of our project. In this module we distribute the generated key to group members. The admin creates the group key as well as private key generation. The admin handles key generation; the admin distributes the key for all group members. The group members are nothing but friends, the group members like college friends, school friends uses this key for security purpose. By this key generation process the hackers can't access our group. The

\section{GROUP KEY GENERATION}

main objective of this project is to be secure messaging inside the group.

\section{CONCLUSION}

Studied a gaggle key agreement drawback, wherever a user is simply attentive to his neighbors whereas the property graph is unfair. additionally, users ar initialized utterly freelance of every different. a gaggle key agreement during this setting is extremely appropriate for applications like social networks. we have a tendency to created 2 passively secure protocols with contributiveness and tested lower Bounds on a spherical complexness, demonstrating that our protocols ar spherical economical. Finally, we have a tendency to created AN actively secure protocol from a passively secure one. In our work, we have a tendency to didn't contemplate a way to update the cluster key a lot of with efficiency than simply running the protocol once more, once user memberships ar dynamical. I have a tendency to aren't clear a way to do that. One will either propose estimations to our present shows or build up another key synchronization with these decisions.

\section{FUTURE ENHANCEMENT}

Likewise, I propose another progress that is veritable secure underneath the starting late formulized Refereed Delegation of Computation model. Finally, I offer raised test results to show the idea of our anticipated progress. The game-plan algorithmic program takes as information a security parameter and yields the general masses key and as such the genius. Note that the star is entire astound at PKG. Session secrets updated supported once cluster admin can end session.

\section{REFERENCES}

1. H. Li, "Learning to rank for information retrieval and natural language processing," Synth. Lect. Human Lang. Technol., vol. 4, no. 1, pp. 1-113,2011.

2. S. Robertson and S. Walker, "Some simple effective approximations tothe 2-poisson model for probabilistic weighted retrieval," in Proc. Annu.Int. ACM SIGIR Conf. Res. Dev. Inf. Retrieval, 1994, pp. 232-241.

3. J. Xu and H. Li, “AdaRank: A boosting algorithm for information retrieval," in Proc.Int.ACMSIGIR Conf. Res. Dev.Inf. Retrieval, 2007,pp. 391-398.

4. D. Cossock and T. Zhang, "Statistical analysis of Bayes optimal subset ranking," IEEE Trans. Inf. Theory, vol. 54, no. 11, pp. 5140-5154,Nov. 2008.

5. T. Liu, "Learning to rank for information retrieval," Found. Trends Inf.Retrieval, vol.3, no. 3, pp. 225-331, 2009.

6. C. Burges, "From RankNet to LambdaRank to LambdaMART:An overview," Microsoft Res., Tech. Rep. MSR-TR-2010-82, 2010.

7. K. Jarvelin and J. Kekalainen, "Cumulated gain-based evaluation of IRtechniques," ACM Trans. Inf. Syst., vol. 20, no. 4, pp. 422-446, 2002.

8. R. Herbrich, T. Graepel, and K.Obermayer, "Large margin rank boundariesfor ordinal regression," in Advances in Large Margin Classifiers. Cambridge, MA, USA: MIT Press, 2000,pp. 115-132. 